\title{
Tendon cell ciliary length as a biomarker of in situ cytoskeletal tensional homeostasis
}

\author{
Michael Lavagnino \\ Keri Gardner \\ Aleksa Michele Sedlak \\ Steven Paul Arnoczky
}

Laboratory for Comparative Orthopaedic Research, College of Veterinary Medicine, Michigan State University, East Lansing, Michigan, USA

Corresponding author:

Steven Paul Arnoczky

Laboratory for Comparative Orthopaedic Research

College of Veterinary Medicine

Michigan State University

East Lansing, Michigan 48824, USA

E-mail: arnoczky@cvm.msu.edu

\section{Summary}

To determine if tendon cell ciliary length could be used as a biomarker of cytoskeletal tensional homeostasis, $20 \mathrm{~mm}$ long adult rat tail tendons were placed in double artery clamps set $18 \mathrm{~mm}$ apart to create a $10 \%$ laxity. The tendons were allowed to contract over a 7 day period under culture conditions. At $0,1,5$, and 7 days the tendon cell cilia were stained and ciliary length measured using confocal imaging. There was a significant $(p<0.001)$ increase in ciliary length at 1 day. At day 5 (when the tendon became visibly taut) there was a significant $(p<0.001)$ decrease in ciliary length compared to day 1. By day 7 the tendon remained taut and ciliary length returned to day zero values $(p=0.883)$. These results suggest that cilia length reflects the local mechanobiological environment of tendon cells and could be used as a potential in situ biomarker of altered cytoskeletal tensional homeostasis.

KEY WORDS: tendon, cilia, recalibration, tensional homeostasis, marker.

\section{Introduction}

Tendon cells are capable of generating a tensional load within their cytoskeleton through an $\alpha$-smooth muscle actin-mediated mechanism, which can generate a considerable traction force against the cell's extracellular matrix ${ }^{1,2}$. This cell based contraction produces a cytoskeletal tensional homeostasis or set point, that calibrates the cell's response to changing extracellular matrix strain through the regulation of gene expression ${ }^{2-4}$. Loss of this cellular tensional homeostasis is thought to occur in tendon cells when collagen fibrils associated with those cells are damaged, or become lax, and lose the ability to bear load $^{5-7}$. This loss of mechanobiological homeostasis has been shown to stimulate catabolic processes within tendon cells ${ }^{5,6,8-10}$ and has been postulated as a causative factor in the development of tendinopathy ${ }^{7}$. The ability to identify in situ alterations in the mechanobiologic environment of tendon samples may provide further insight into the role of mechanobiology in the development and progression of tendinopathy. Our lab has demonstrated that loss of tensional homeostasis in tendon cells is accompanied by an increase in the length of the cells' primary cilia ${ }^{11}$. Primary cilia are sensory organelles which detect and transmit mechanical and chemical information between the extracellular environment and the cell12-16. Tendon cell cilia have been shown to adapt their length to changes in their local loading environments; increasing their length in response to decreased mechanobiologic stimulus and shortening to their resting homeostatic length following application of externally applied cyclic loads ${ }^{11}$. However, the ability of intrinsically generated (i.e. cell-based) tensional homeostasis to regulate cilial length in tendon cells is unknown.

Therefore, the purpose of the current study was to determine if elongated tendon cell cilia in lax rat tail tendon fascicles (RTTfs) can be returned to control lengths through a previously described actin-based cellular contraction mechanism ${ }^{3}$. A previous study has demonstrated that tendon cells are able to actively contract RTTfs with a $10 \%$ laxity over a 7 day period and recover normal tendon cell tensional homeostasis as determined by inhibition of collagenase synthesis ${ }^{3}$. In the current study, we hypothesize that the primary cilia of RTTfs tendon cells will significantly increase in length following creation of a $10 \%$ laxity in the tendon. In addition, we hypothesize that the length of preliminary cilia will return to control values following the cellbased contraction of the tendon over 7 days and the return of the previously documented tensional homeostasis. Validation of these hypotheses could support the validity of cilial length as a potential biomarker of the in situ mechanobiologic status of tendon cells.

\section{Methods}

Following Institutional Animal Care and Use Committee approval, tendons were obtained from the tails of 
adult 3-month-old Sprague-Dawley rats immediately after euthanasia and maintained in DMEM supplemented with $10 \%$ FBS, $1 \%$ antibiotic/antimycotic and $0.75 \%$ ascorbic acid while incubated at $37^{\circ} \mathrm{C}$ and $10 \%$ $\mathrm{CO}_{2}$. Twenty-four rat tail tendon fascicles (RTTfs) (20 $\mathrm{mm}$ long) from each of 5 animals were placed in double artery clamps set $18 \mathrm{~mm}$ apart so as to create a grip to grip laxity of $10 \%$ of total RTTfs length. The RTTfs were divided evenly into groups and analyzed at one of four different time periods as follows: fresh control (Day 0 ) or allowed to freely contract between the clamps for 1 day (Day 1), 5 days (Day 5), or 7 days (Day 7). A previous study investigated the re-establishment of tensional homeostasis in lax tendons utilizing younger 1 -month-old rat tail tendons at 3 and 5 days $^{3}$. The tendons used in this study were from older 3-month-old rats that have been shown to have a slower rate of cell-based tendon contraction than 1month-old rats ${ }^{17}$. Therefore, in the current study the later time points of 5 and 7 days were used as this corresponds to when the tendons became taut between the clamps for 3-month-old rats.

One centimeter segments from each of the six RTTfs were harvested per treatment group and stained with 250 nM Tubulin Tracker Green (Life Technologies) for 30 minutes at $37^{\circ} \mathrm{C}$. Stained RTTs were washed vigorously in dPBS for 30 minutes, then placed into two subsequent non-vigorous dPBS washes for a total of 3-5 hours of washing. Cilia were visualized using Pro Long Gold with DAPI (Life Technologies) and confocal images were created using an Olympus Fluo view 1000 laser scanning microscope. The length of each primary cilia, from the centriole to the tip of the ciliary axenome, was measured using Image $\mathrm{J}$ as previously performed ${ }^{11}$. Cilia lengths were presented as the average and standard deviation of the 5 rats and analyzed using a two factor ANOVA and Tukey's post hoc test with significance set at $p<0.05$.

\section{Results}

The average length of the primary cilia in tendon cells of fresh (Day 0) rat tail tendon fascicles (RTTfs) was $1.35 \pm 0.11 \mu \mathrm{m}$. One day following creation of a $10 \%$ laxity in their grip to grip length the RTTfs remained visibly lax and the length of the primary cilia significantly increased $(p<0.001)$ to $2.76 \pm 0.19 \mu$ m (Figs. 1, 2). By day 5 the RTTfs appeared visually taut between the clamps and the cilia had significantly

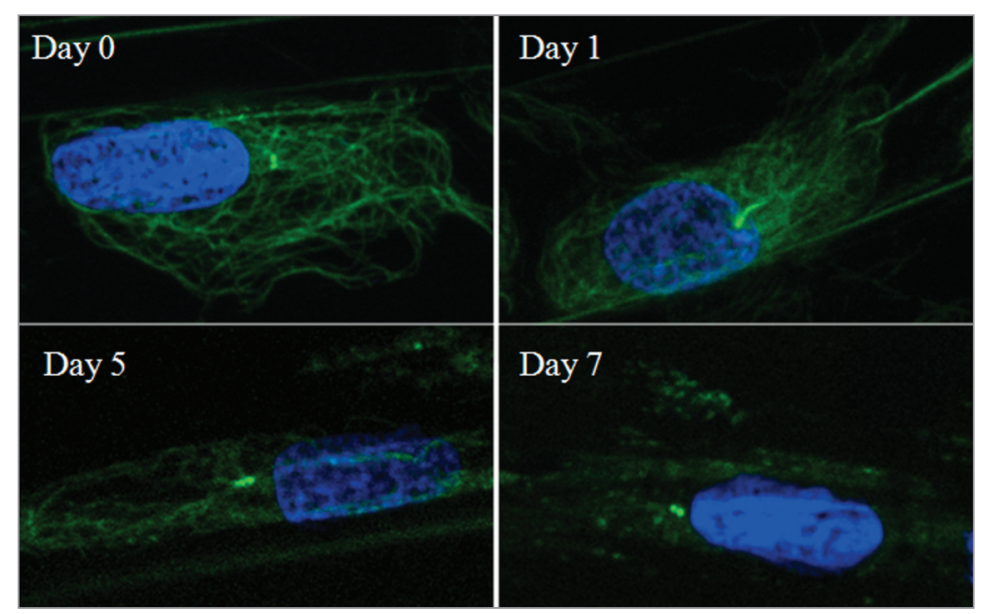

Figure 1. Confocal images of in situ tendon cells with associated nuclei (DAPI) and cilia (Tubulin Tracker Green) from tendons allowed to freely contract between clamps at Day 0, Day 1, Day 5, and Day 7 with the tendon remaining lax at Day 1 , but visibly taut at Day 5 and Day 7.

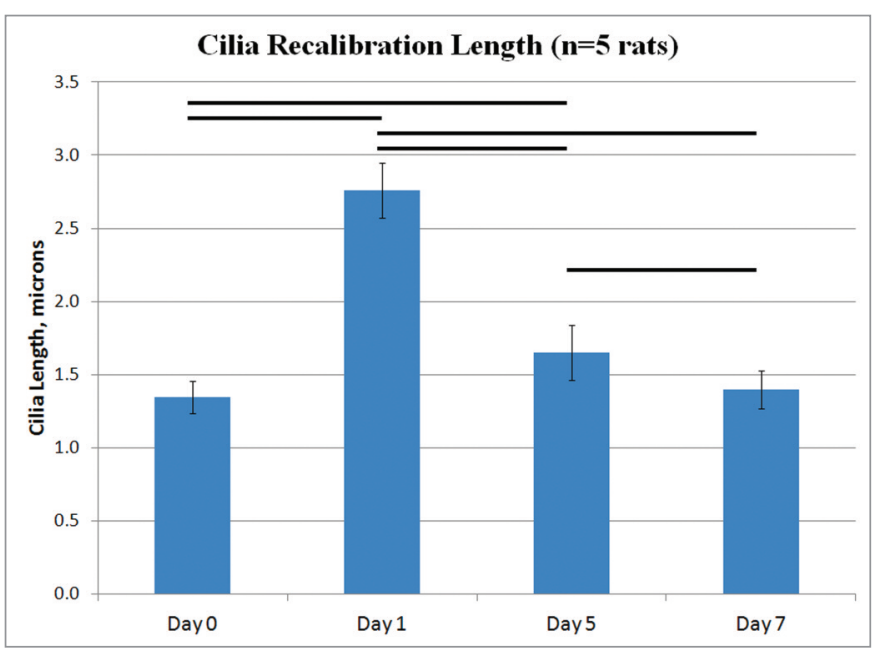

Figure 2. Graph showing the average cilia length of in situ tendon cells from tendons allowed to freely contract between clamps at A) Day 0, B) Day 1, C) Day 5, and D) Day 7 with the tendon remaining lax at Day 1 , but visibly taut at Day 5 and Day 7 . Lines above bars indicate significantly different values, $p<0.05$. 
$(p<0.001)$ shortened $(1.65 \pm 0.19 \mu \mathrm{m})$ with respect to day 1 lengths. The RTTfs remained taut at day 7 and had an average length of $1.40 \pm 0.13 \mu \mathrm{m}$ which was not significantly $(p=0.883)$ different from 0 day (fresh) samples (Figs. 1, 2).

\section{Discussion}

The ability of tendon cells to maintain their normal cytoskeletal tensional homeostasis has been shown to play a critical role in regulating catabolic gene expression and protein synthesis $2,5-8,10$. Loss of cytoskeletal homeostasis may occur as a result of an absence of the normal, residual tension in the pericellular matrix of tendons and ligaments caused by injury or repetitive exercise ${ }^{18-20}$. Such alterations in the local mechanobiologic environment of tendon cells have been implicated as the inciting factor in the pathogenesis of tendinopathy ${ }^{7,21}$. However, since an upregulation of catabolic factors associated with tendinopathy can also be triggered by other humoral stimuli (i.e. IL-1b, PGE2) 22-24 a biomarker which specifically reflects the local, in situ mechanobiologic status of the cell could be helpful in supporting this theory. The primary cilia of tendon cells have been shown to adapt their length to changes in their local loading environments; increasing their length in response to decreased mechanobiologic stimulus and shortening to their resting homeostatic length following application of externally applied cyclic loads ${ }^{11}$.

The results of the current study demonstrate that cilia elongate within 24 hours following induced loss of normal cytoskeletal tension through tendon laxity. This outcome confirms a previous study which also demonstrated cilia elongation with tendon laxity ${ }^{11}$. In addition, the same previous study also documented that with continuous loss of cytoskeletal tension, tendon cilia length does not change significantly after the initial 24 hour lengthening ${ }^{11}$. This "set control length" has also been demonstrated in chondrocyte cilia $^{12}$ suggesting that the average cilia length would not increase beyond this set length once cytoskeletal tension has been lost. This growth in ciliary length has also been documented in other tissues that have lost external stimuli or internal tension ${ }^{25-28}$. The precise reason for primary cilia to lengthen in the absence of physical stimuli is unclear. However, it may represent an attempt by the tendon cell to 'amplify' the transmission of weakened mechanotransduction signals ${ }^{11,29}$.

Externally applied forces transmitted through the extracellular matrix have been shown to reverse cilia elongation both in tendon ${ }^{11}$ and other tissues ${ }^{25-}$ $27,29,30$. However, the current study is unique as it demonstrates that cilia elongation can also be reversed through internally generated cell traction. This actin-mediated cellular force contracts the extracellular matrix to eliminate the laxity in the extracellular matrix and allows the tendon cells to regain their normal internal tensional homeostasis ${ }^{3}$. Previous studies from our lab have documented a similar contraction pattern of lax RTTfs and a return to cellular homeostasis between 5 and 7 days, as manifested by an inhibition of interstitial collagenase synthesis ${ }^{3,17}$.

The current study demonstrates that as the cellular tensional homeostasis is restored between 5 and 7 days, the length of the cilia decreases until it returns to a length not statistically different than control values. Since cilial length is reflected by the local mechanobiologic environment of the cell, the findings of the current study suggest that increased cilial length may be an effective biomarker with which to document alterations in the in situ mechanobiologic signaling environment of tendon cells. The histological assessment of cilial length in histologic sections of tendinopathic tisues may help clarify the potential role of mechanobiologic understimulation $^{7}$ in the etiopathogenesis of the catabolic changes associated with tendinopathy ${ }^{31}$.

\section{Conclusion}

In conclusion, the current study demonstrates the relationship of tendon laxity and tendon cell tensional homeostasis to the length of their primary cilia. In addition, results support the potential use of tendon cell ciliary length as a biomarker of the in situ mechanobiologic environment of tendon cells.

\section{References}

1. Brown RA, Prajapati R, McGrouther DA, Yannas IV, Eastwood M. Tensional homeostasis in dermal fibroblasts: mechanical responses to mechanical loading in three-dimensional substrates. J Cell Physiol 1998;175(3):323-332.

2. Lavagnino M, Arnoczky SP. In vitro alterations in cytoskeletal tensional homeostasis control gene expression in tendon cells. J Orthop Res 2005; 23(5):1211-1218.

3. Gardner K, Lavagnino M, Egerbacher M, Arnoczky SP. Reestablishment of cytoskeletal tensional homeostasis in lax tendons occurs through an actin-mediated cellular contraction of the extracellular matrix. J Orthop Res 2012 30(11):1695-1701.

4. Tresoldi I, Oliva F, Benvenuto M, Fantini M, Masuelli L, Bei $\mathrm{R}$, Modesti A. Tendon's ultrastructure. Muscles Ligaments Tendons Journal 2013; 3(1):2-6.

5. Lavagnino M, Arnoczky SP, Egerbacher M, Gardner KL, Burns ME. Isolated fibrillar damage in tendons stimulates local collagenase mRNA expression and protein synthesis. J Biomech 2006; 39(13):2355-2362.

6. Arnoczky SP, Lavagnino M, Egerbacher M, Caballero O, Gardner K, Shender MA. Loss of homeostatic strain alters mechanostat "set point" of tendon cells in vitro. Clin Orthop Relat Res 2008; 466(7):1583-1591.

7. Arnoczky SP, Lavagnino M, Egerbacher M. The mechanobiological aetiopathogenesis of tendinopathy: is it the over-stimulation or the under-stimulation of tendon cells? Int J Exp Pathol 2007; 88(4):217-26.

8. Arnoczky SP, Tian T, Lavagnino M, Gardner K. Ex vivo static tensile loading inhibits MMP-1 expression in rat tail tendon cells through a cytoskeletally based mechanotransduction mechanism. J Orthop Res 2004; 22(2):328-333.

9. Egerbacher M, Arnoczky SP, Caballero O, Lavagnino M, Gardner KL. Loss of homeostatic tension induces apopto- 
sis in tendon cells: an in vitro study. Clin Orthop Relat Res 2008; 466(7):1562-1568.

10. Lavagnino M, Arnoczky SP, Tian T, Vaupel Z. Effect of amplitude and frequency of cyclic tensile strain on the inhibition of MMP-1 mRNA expression in tendon cells: an in vitro study. Connect Tissue Res 2003; 44(3-4):181-187.

11. Gardner K, Arnoczky SP, Lavagnino M. Effect of in vitro stress-deprivation and cyclic loading on the length of tendon cell cilia in situ. J Orthop Res 2011; 29(4):582-587.

12. Donnelly $E$, Williams $R$, Farnum $C$. The primary cilium of connective tissue cells imaging by multiphoton microscopy. Anat Rec 2008; 291:1062-1073.

13. McGlashan SR, Knight MK, Chowdhury TT, et al. Mechanical loading modulates chondrocyte primary cilia incidence and length. Cell Biol Int 2010; 34:441-446.

14. Farnum CE, Williams RM, Donnelly E. Analyzing primary cilia by multiphoton microscopy. Methods Cell Biol 2009; 94:117-135

15. Delaine-Smith RM, Reilly GC. Mesenchymal stem cell responses to mechanical stimuli. Muscles Ligaments Tendons Journal 2012; 2(3):169-180.

16. Docking S, Samiric T, Scase E, Purdam C, Cook J. Relationship between compressive loading and ECM changes in tendons. Muscles Ligaments Tendons Journal 2013;3(1):7-11.

17. Lavagnino M, Gardner K, Arnoczky SP. Age-related changes in the cellular, mechanical, and contractile properties of rat tail tendons. Connect Tissue Res 2013; 54(1):70-75.

18. Crisco JJ, Chelikani S, Brown RK, et al. The effects of exercise on ligamentous stiffness in the wrist. J Hand Surg 1997; 22A:44-48.

19. Grana WA, Muse $G$. The effect of exercise on laxity in the anterior cruciate ligament deficient knee. Am J Sports Med 1988;16:586-588.

20. Vesentini S, Redaelli A, Gautieri A. Nanomechanics of collagen microfibrils. Muscles Ligaments Tendons Journal 2013; 3(1):23-24.
21. Birch HL, Thorpe CT, Rumian AP. Specialization of extracellular matrix for function in tendons and ligaments Muscles Ligaments Tendons Journal 2013; 3(1):12-22.

22. Yang G, Im HJ, Wang JH. Repetitive mechanical stretching modulates IL-1beta induced COX-2, MMP-1 expression, and PGE2 production in human patellar tendon fibroblasts. Gene 2005; 363:166-72.

23. Alvares O, Klebe R, Grant G, Cochran DL. Growth factor effects on the expression of collagenase and TIMP-1 in periodontal ligament cells. J Periodontol 1995; 66:552-558.

24. Dakin SG, Dudhia J, Werling NJ, Werling D, Abayasekara DR, Smith RK. Inflamm-aging and arachadonic acid metabolite differences with stage of tendon disease. PLoS One 2012; 7(11):e48978.

25. Wang L, Weidenfeld R, Verghese E, et al. Alterations in renal cilium length during transient complete ureteral obstruction in the mouse. J Anat 2008; 213:79-85.

26. Verghese E, Weidenfield R, Bertram JF, et al. Renal cilia display length alterations following tubular injury and are present early in epithelial repair. Nephrol Dial Transplant 2008; 23:834-841.

27. Verghese E, Ricardo DS, Weidenfield R, et al. Renal primary cilia lengthen after acute tubular necrosis. J Am Soc Nephrol 2009; 20:2147-2153.

28. Kim J, Lee JE, Heynen-Genel S, et al. Functional genomic screen for modulators of ciliogenesis and cilium length. $\mathrm{Na}$ ture 2010; 464:1048-1052.

29. Donnelly E, Ascenzi M-G, Farnum C. Primary cilia are highly oriented with respect to collagen direction and long axis of extensor tendon. J Ortho Res 2010; 28:77-82.

30. Resnick A, Hopfer U. Force-response consideration in ciliary mechanosensation. Biophys J 2007; 93:1380-1390.

31. Jelinsky SA, Rodeo SA, Li J, Gulotta LV, Archambault $\mathrm{JM}$, Seeherman HJ. Regulation of gene expression in human tendinopathy. BMC Musculoskelet Disord 2011; 12:86. 